\title{
Feature Error Model for Integrity of Pattern-based Visual Positioning
}

\author{
Chen Zhu, Christian Steinmetz, Boubeker Belabbas, and Michael Meurer \\ Institute of Communications and Navigation, \\ German Aerospace Center (DLR), Oberpfaffenhofen, Germany \\ Email: \{Chen.Zhu, Christian.Steinmetz, Boubeker.Belabbas, Michael.Meurer\}@dlr.de
}

\section{BIOGRAPHY}

Chen Zhu is a full-time research fellow at the Institute of Communications and Navigation, German Aerospace Center (DLR) since April 2018, after submitting his Ph.D. dissertation at Technical University of Munich, in Munich, Germany. He received his B.Sc. in Automation Engineering from Tsinghua University, in Beijing, China in 2009, and his M.Sc. in Communications Engineering in 2011 from Technical University of Munich. His research interests include visual navigation, multi-sensor fusion, robotic swarm navigation, and he is focusing on the integrity of the techniques.

Christian Steinmetz obtained a Bachelor of Science degree in Electrical Engineering and Information Technology at the Technical University of Munich. He is currently studying the consecutive Master's program there. For his Master's thesis, he joined the Department of Navigation at the Institute of Communications and Navigation, German Aerospace Center (DLR).

Boubeker Belabbas obtained a MSc. Degree in Mechanical Engineering from ENSEM in Nancy (France) and a specialized MSc. Degree in Aerospace Mechanics from SUPAERO in Toulouse (France). He joined DLR in 2001 and developed the navigation integrity activities. He now leads the GNSS integrity team within the Department of Navigation at the Institute of Communications and Navigation. His field of competence includes GNSS with augmentations (SBAS, GBAS), Advanced Receiver Autonomous Integrity Monitoring, GNSS/INS Hybridization with integrity, and Alternative Positioning Navigation and Timing.

Prof. Michael Meurer is the director of the Department of Navigation at the German Aerospace Center (DLR) and of the Center of Excellence for Satellite Navigation. In addition, he is a full professor of electrical engineering and director of the Chair of Navigation at the RWTH Aachen University. His current research interests include GNSS signals, GNSS receivers and navigation for safety-critical applications.

\footnotetext{
ABSTRACT

Camera-based visual navigation techniques can provide high precision infrastructure-less localization solutions using visual patterns, and play an important role in the environments where satellite navigation has significantly degraded performance in availability, accuracy, and integrity. However, the integrity monitoring of visual navigation methods is an essential but hardly-solved topic, since modelling the geometric error for cameras is rather challenging. This work proposes a highprecision geometric error model of detected feature corners for chessboard-like patterns. The model is named as Chessboard Corner Geometric Error Model (CCGEM). By applying the model to images containing chessboard-like patterns, the extracted corner location accuracy can be predicted in different lighting conditions. The coefficients in the model can be adapted to each distinct camera-lens system through a calibration process. The proposed method first models the intensity distribution in the local neighboring area of the extracted corner by taking the raw image as measurement input. Then, the geometric error of the feature location is modelled as a function of the distribution parameters. We show that the model fits the measurement error well in both simulated and real images. The proposed CCGEM also provides a conservative fitting model with risk probability information, which can be applied in the integrity monitoring of vision-based positioning.
} 


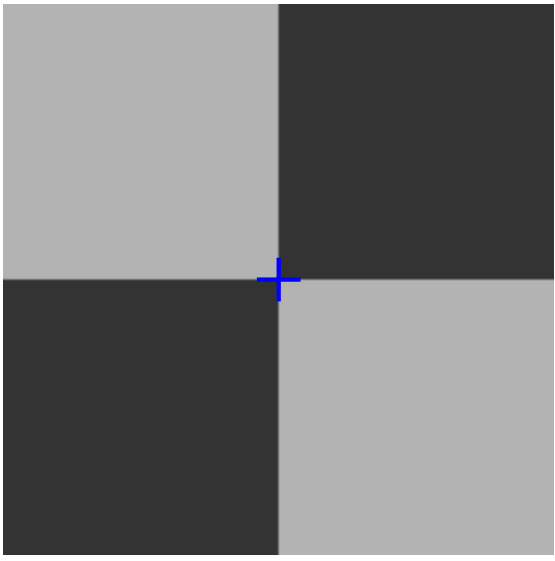

(a) Feature extraction without noise

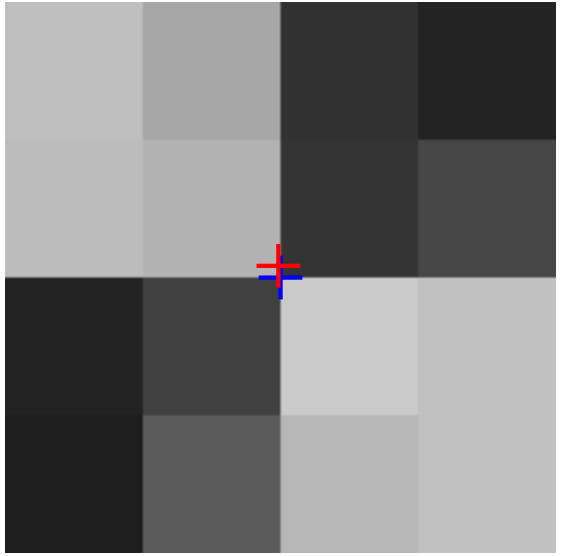

(b) Feature extraction with noise

Figure 1: Photometric error and consequential geometric error in feature extraction

\section{INTRODUCTION}

Camera-based visual positioning has been widely investigated for autonomous landing of unmanned aerial vehicles (UAV) using a designed pattern as a landing pad. For instance, the approaches from Sharp et al. [1] and Cesetti et al. [2] have attracted great attentions of the research community. In addition, visual navigation techniques have huge potential in various applications, especially in environments such as urban areas where satellite navigation may have significantly degraded performance due to lacking of signal availability and multipath effects, e.g., as shown in the work from Narula et al. [3]. However, quantitative integrity monitoring of visual navigation is not yet a well-solved problem.

Three basic components are essential for developing the visual navigation integrity. First of all, a feature location error model in nominal situations is required. Second, the dilution of precision (DOP) needs to be calculated to evaluate the geometric impact on the estimated position using cameras. Last but not least, specific fault detection and exclusion (FDE) schemes should be developed for different fault modes in visual navigation integrity monitoring. This work focuses on the development of a stochastic error model for the feature location. A stochastic error model is not only required for monitoring the nominal performance of the visual navigation methods, but can also give researchers a better understanding of the error sources in the vision measurements, so that the fault modes can be defined appropriately.

Meanwhile, the characterization of the error in the extracted feature locations is however one of the largest difficulties towards vision integrity monitoring. In feature-based visual navigation methods, the coordinates of the 2D features are used as sensor measurements. However, the coordinates are indirect measurements. For camera sensors, the raw measurements are image pixel intensity values. The measurement noise of pixel intensities is normally referred to as photometric error $n_{I}$, which is modeled as a zero-mean Gaussian distribution with covariance $\sigma_{n_{I}}$. Fig. 1 illustrates the photometric noise and its impact on the feature extraction using a simple instance. Fig. 1a shows a noise-free chessboard image, and the blue "+" marker denotes the ground truth position of the corner point. In Fig. 1b, the existence of photometric noise results in the slight variation of the black and white colors. Consequently, the corner location extracted by a feature detector, which is indicated by the red "+" marker, is also erroneous compared to the ground truth. The error of the estimated feature location is referred to as geometric error.

There are several challenges towards a general stochastic geometric error model. First, the distribution of feature points is not homogeneous, i.e., different feature points may follow distinct distributions. The lighting condition determines the intensity values, and the feature type as well as the view point influences the geometric distribution of the intensities around the feature. Either impact results in changes of the geometric error distribution. Moreover, since the feature extraction algorithms normally contain complicated and heuristic operations, describing the error transformation from photometric error in intensity domain to geometric error in feature location domain is rather challenging. In addition, the physical optical systems also have impacts on the measurement images. There is optical blur due to effects like diffraction and diffusion when the light rays pass through the lenses, which is normally described by a Gaussian point spread function (PSF) [4]. As a result, the distribution of the feature location error is dependent on the camera and lens applied. Due to the diversity in the geometric error distribution, it is not reasonable to simply build statistics from huge amount of data to derive a homogeneous distribution as the error model. 


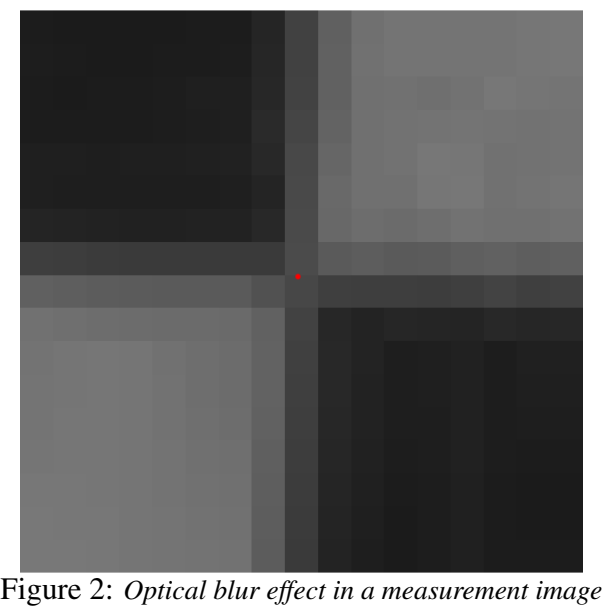

Though the feature geometric error distributions are necessary in visual navigation, the aforementioned challenges are not well solved yet. In state-of-the-art visual navigation methods such as ORB-SLAM [5], the geometric error covariance is normally chosen by using some heuristic values (e.g., set as 1 pixel in ORB-SLAM). This is unacceptable for integrity monitoring, since tuning the parameter for specific scenarios does not ensure that the model is valid if the visible scene changes. Reprojection error (feature location residual given the estimated pose) is widely used in visual navigation textbooks such as [6] to describe the feature error. However, the statistics of residuals is obviously not a proper error model, since the estimated states used for calculating the reprojection error can already be biased. Kumar and Osechas have shown in [7], and Edwards et al. have shown in [8] that for designed patterns, the feature location error follows a Gaussian distribution in nominal situations. Nevertheless, the results are still qualitative, since the variance of the distribution is still an ad-hoc value obtained from experiments for particular scenarios.

In this work, we propose a subpixel-precision geometric error model of detected corners, named as CCGEM (Chessboard Corner Geometric Error Model). CCGEM targets at a specific type of corners (chessboard-like 'X'-junctions), which can either be from a designed landmark or be extracted natural features. It models the stochastic geometric error as a function of a few local parameters of the measurement image, which varies as the lighting or the visible scene changes. The parameters can be extracted from the local image patches around the corners with affordable complexity. Some coefficients in the model are dependent on the exploited optical instrument. These coefficients can be obtained through a calibration process for each distinct camera-lens combination, so that it is generalizable for different optical systems from end users. Therefore, CCGEM is a quantitative error model of the feature location which is generalizable for different optical systems and lighting conditions. In addition, conservative strategies are proposed in the coefficients fitting process for integrity demands.

\section{SYSTEM MODEL}

In this section, we clarify the system model and the assumptions in developing the error model. A measurement image from a digital camera can be modelled as follows:

$$
I=I_{0} \star G+n_{I} \in \mathbb{Z}_{I}^{N_{h} \times N_{w}}
$$

where $N_{h}$ and $N_{w}$ are the height and width of the images respectively, and $\mathbb{Z}_{I}$ is the set of intensity values. For a common 8-bit digital image, $\mathbb{Z}_{I}=\{z \mid z=0,1, \ldots, 255\}$. For generality, in this paper we use normalized intensities with discrete values in domain $[0,1]$ without specifying the quantization level. $I_{0}$ denotes the image corresponding to the true luminance of the visible scene during the exposure time. Following the convention, the impact from the optical system can be modelled as the convolution of $I_{0}$ with a two dimensional digital Gaussian filter $G$. As an intuitive illustration, Fig. 2 demonstrates the optical blur effect in a real measurement image. $n_{I}$ is the Gaussian photometric noise.

For an extracted feature point $u \in \Omega \subset \mathbb{R}^{2}$ from a measurement image $I$ ( $\Omega$ denotes the image plane), the estimated location of the feature $\hat{u}$ is erroneous compared with the true value. Since we focus on the stochastic error of the feature location measurements in this work, the measurement bias from specific feature detectors is not discussed here. Nevertheless, the stochastic error model can be used in statistical tests to identify if a feature location measurement is biased or not. 


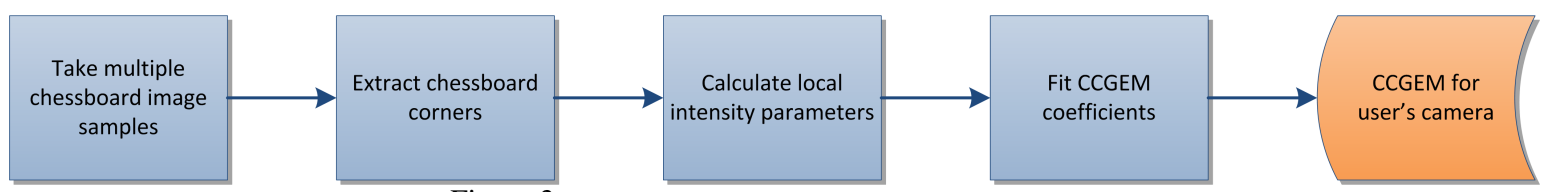

Figure 3: Work flow of generating CCGEM for a camera.

Consequently, in nominal cases, the extracted feature location can be written as

$$
\hat{u}=F(I)=u+n_{u}
$$

According to the earlier work from Kumar et al. [7] and Edwards et al. [8], the geometric error follows a two dimensional Gaussian distribution in the constant lighting environment, and the two dimensions are isotropically distributed as long as the camera is stable, so that the covariance of the extracted feature location error can be represented as

$$
\Sigma_{u}=E\left\{n_{u} n_{u}^{T}\right\}=\left[\begin{array}{cc}
\sigma_{x}^{2} & \sigma_{x y} \\
\sigma_{x y} & \sigma_{y}^{2}
\end{array}\right]=\left[\begin{array}{cc}
\sigma_{u}^{2} & 0 \\
0 & \sigma_{u}^{2}
\end{array}\right] .
$$

As a result, the stochastic geometric error of features can be sufficiently described by the parameter $\sigma_{u}$. However, as mentioned in the introduction section, modelling the value of $\sigma_{u}$ is challenging, since the error distribution varies with the local intensities, which is influenced by feature types, lighting conditions, and distinct optical systems.

We simplify the problem by only investigating a particular type of features, i.e., the chessboard-like ' $\mathrm{X}$ '-junction corners as shown in Fig. 1. There are a few favorable properties of ' $X$ '-junctions for geometric error modelling. First, by only detecting the ' $X$ '-junctions, the error distribution variation introduced by the diversity of the corner shape can be solved. Second, the neighbor area of a chessboard-like corner is geometrically symmetric, and the corner is located at the crossing of the two lines. As a result, it is feasible to find a reliable unbiased estimator to locate the corner. In our approach, we apply the stateof-the-art saddle point estimator [9], which is designed specifically for ' $\mathrm{X}$ '-junctions with subpixel precision. Moreover, it is possible to describe the geometric distribution of the intensities around the corner with only a few parameters, which makes our proposed error model adaptive to lighting and optical changes, while keeping it non-exhaustive and computationally feasible.

\section{WORK FLOW OF APPLYING CCGEM}

In this section, we provide a summary of the CCGEM as well as the work flow of generating and applying the model for end users. The detailed explanation and derivation of the proposed error model will be provided in the following sections.

For chessboard-like features (' $\mathrm{X}$ '-junctions), we propose a general geometric error model named CCGEM in this work considering the aforementioned impacts. The model is summarized as

$$
\sigma_{u}=g\left(\sigma_{I}, \Delta I, s_{L}\right)=\left(\alpha_{1}+\alpha_{2} s_{L}^{\alpha_{3}}\right) \frac{\sigma_{I}}{\Delta I}
$$

where $\sigma_{u}$ is the standard deviation of the stochastic error in the extracted feature location defined in Eqn. (3), which is dependent on the local photometric error covariance $\sigma_{I}$, the local intensity difference parameter $\Delta I$, and the lens blurring parameter $s_{L}$. All the three parameters are extracted from the neighbor region of the detected corners in the measurement images. $\alpha_{1}, \alpha_{2}$, and $\alpha_{3}$ are coefficients of the CCGEM model, which can be estimated adaptively through a calibration process and do not change for different measurement images. As a result, once the coefficients are generated, the model can be applied for the same optical system in changing environments to predict the covariance of the feature location error. When exploiting the model, only the measurement image is needed as input.

Fig. 3 demonstrates the work flow of generating CCGEM for a specific optical system in an offline calibration process. First of all, a large set of static images of a checkerboard needs to be taken in a controlled environment with consistent lighting in order to calibrate the coefficients for a distinct camera-lens system. The images have the same visible scene but independent photometric noise. By extracting the chessboard corners from all the images using the subpixel precision corner detector in [9], the covariance of each corner location $\varsigma_{u}$ can be obtained from the statistics of $\hat{u}$. Since the feature detection algorithm is not the main focus of this work, we assume the chessboard corners can be correctly detected. For each corner point, we 


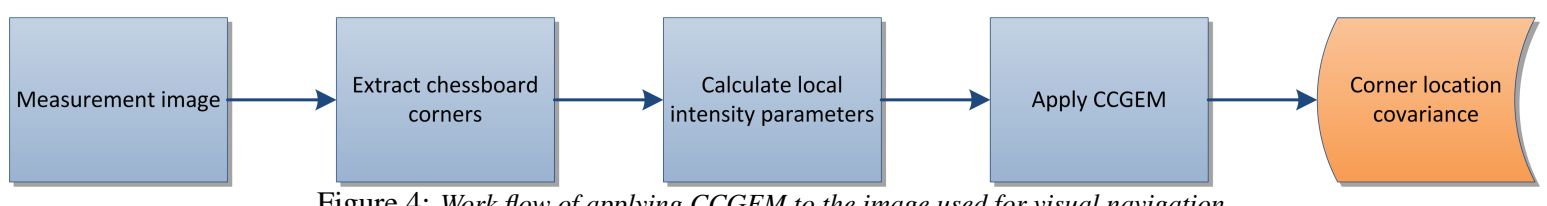

Figure 4: Work flow of applying CCGEM to the image used for visual navigation.

first calculate the intensity histogram at the neighbor area of the extracted corner from the raw image. Then, the histogram is fitted with a Gaussian mixture distribution model. Since a chessboard-like pattern contains distinguishable black and white pixels around a corner area, the Gaussian mixture model (GMM) includes a component distribution from the white areas and another component from black areas. The mean and covariance of the two Gaussian components can be estimated from the Gaussian mixture fitting. From the fitted GMM, the parameter $\sigma_{I}$ and $\Delta I$ can be extracted. Moreover, with the parameters of the Gaussian components, the parameter $s_{L}$, which is a metric of the optical blurring impact introduced by the lens, can be estimated. Consequently, the coefficients $\alpha_{1}, \alpha_{2}$, and $\alpha_{3}$ in Eqn. (4) can be calculated by curve fitting exploiting the measurements from all the extracted chessboard corner points. According to applications, the curve fitting may follow different criteria. We provide both a least-squares fitting strategy and a conservative fitting strategy for critical applications like integrity monitoring.

After the offline calibration of the coefficients, the model can be applied to the onboard measurement images. Fig. 4 demonstrates the work flow of applying CCGEM to images containing chessboard-like ' $X$ '-junctions. With an online processed image, the stochastic geometric error can be predicted by applying the model with local intensity parameters of the corner $\left(\sigma_{I}, \Delta I\right.$, and $\left.s_{L}\right)$, which are extracted with the same procedure as in the calibration.

As a result, the feature location error for images containing chessboard-like patterns can be modelled by the proposed CCGEM, which can be used for applications such as integrity monitoring of the applied visual navigation method.

\section{CHESSBOARD CORNER GEOMETRIC ERROR MODEL}

Almost all the corner detectors exploit the fact that there would be a significant gradient of intensity values in both dimensions around a corner. Generally we can model a corner detector as

$$
\hat{u}=F(I, \nabla I),
$$

where $I$ is the image patch around the corner and $\nabla I$ is the corresponding gradient of intensities. For a chessboard ' $\mathrm{X}$ 'junction corner, the effective image gradients around the corner are obtained from the subtraction between a white pixel and a black pixel. Without loss of generality, we assume the black and white pixel intensities follow independent distributions. In absence of measurement noise, the probability density function (pdf) of the intensity around an ideal chessboard corner consists of two dirac functions at the black and white color respectively. Fig. 5 shows the distribution of the noise-free image in Fig. 1a as a simple example.

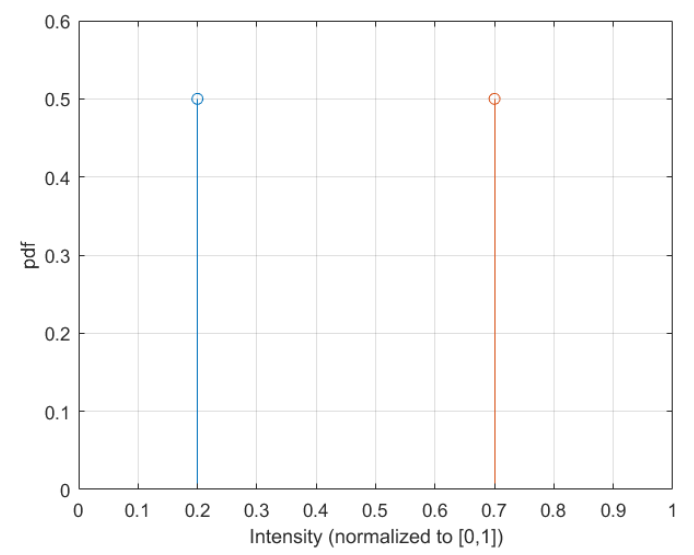

Figure 5: Probability density function of intensity in noise free case

With the Gaussian measurement noise ( $n_{I}$ in Eqn. (1)), the intensity distribution becomes a Gaussian mixture distribution, 
as shown in Fig. 6 (corresponding to the image in Fig. 1b).

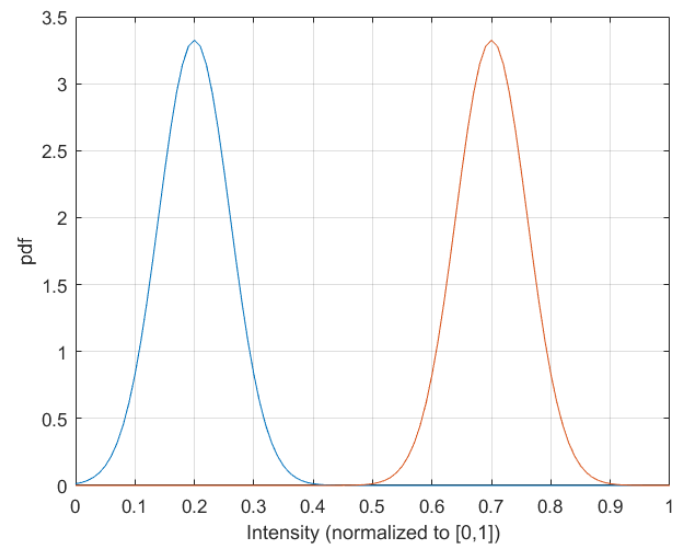

Figure 6: Probability density function of intensity with Gaussian noise

To keep the model general, the noise covariances for different colors are allowed to be different. The black pixels follow the Gaussian distribution $I_{b} \sim \mathscr{N}\left(\bar{b}, \sigma_{b}^{2}\right)$, while the white pixels follow $I_{w} \sim \mathscr{N}\left(\bar{w}, \sigma_{w}^{2}\right)$. The corner detector exploits significant gradients formed by a pair of white pixel and black pixel. Hence in either $x$ or $y$ dimension, the effective gradient contributing to the corner detection is generally calculated as $\nabla I=I_{w}-I_{b}$. (In practice, it is normally done by filtering the image with a 2D digital gradient filter.) Consequently, the image gradient utilized for extracting chessboard corners follows a Gaussian distribution

$$
\nabla I \sim \mathscr{N}\left(\Delta I, \sigma_{I}^{2}\right), \quad \text { where } \Delta I=\bar{w}-\bar{b}, \sigma_{I}=\sqrt{\sigma_{w}^{2}+\sigma_{b}^{2}} .
$$

Therefore, the covariance of the feature geometric error depends on the two distribution parameters $\sigma_{I}$ and $\Delta I$.

It can be proved that for the applied chessboard corner extraction method [9], the following relation holds:

$$
\sigma_{u} \sim \frac{\sqrt{\sigma_{w}^{2}+\sigma_{b}^{2}}}{\bar{w}-\bar{b}}=\frac{\sigma_{I}}{\Delta I},
$$

i.e., the uncertainty of the corner location estimation $\sigma_{u}$ is linearly proportional to the uncertainty of the intensity difference between the two colors, and reversely proportional to the mean difference. Intuitively, if the black and white components are more distinguishable (higher contrast), the extracted corner location is more accurate. If the photometric measurement noise is larger, it is more difficult to distinguish the two colors, which results in larger corner location uncertainty.

In order to model the geometric error for a specific corner, the two distribution parameters need to be extracted from the measurement image. We use the Gaussian mixture model (GMM) to demonstrate the intensity distribution for each extracted chessboard corner feature. Given a corner detected from a noisy measurement image, an intensity histogram of the neighboring region can be built. By applying Gaussian mixture fitting to the histogram, the parameters of the generated GMM $\hat{\bar{w}}, \hat{\bar{b}}, \hat{\sigma}_{w}$ and $\hat{\sigma}_{b}$ can be obtained. Subsequently, the intensity gradient distribution parameters can be calculated as

$$
\widehat{\Delta I}=\hat{\bar{w}}-\hat{\bar{b}},
$$

and

$$
\hat{\sigma}_{I}=\sqrt{\hat{\sigma}_{w}^{2}+\hat{\sigma}_{b}^{2}}
$$

The local intensity distribution GMM fitting is tested both on simulated images, shown in Fig. 7, and on real images, shown in Fig. 8. In the simulation, noise added on black and white colors are with different standard deviation, so that the performance of the fitting is verified for general cases. In real images, the noise variances of the two colors should be almost the same in a local image patch.

According to the fitting results, the model represents the local intensity distribution well. However, it can be seen from the Fig. 8 that there is a gray zone between the black- and white-component Gaussian peaks in the real measurement images. 

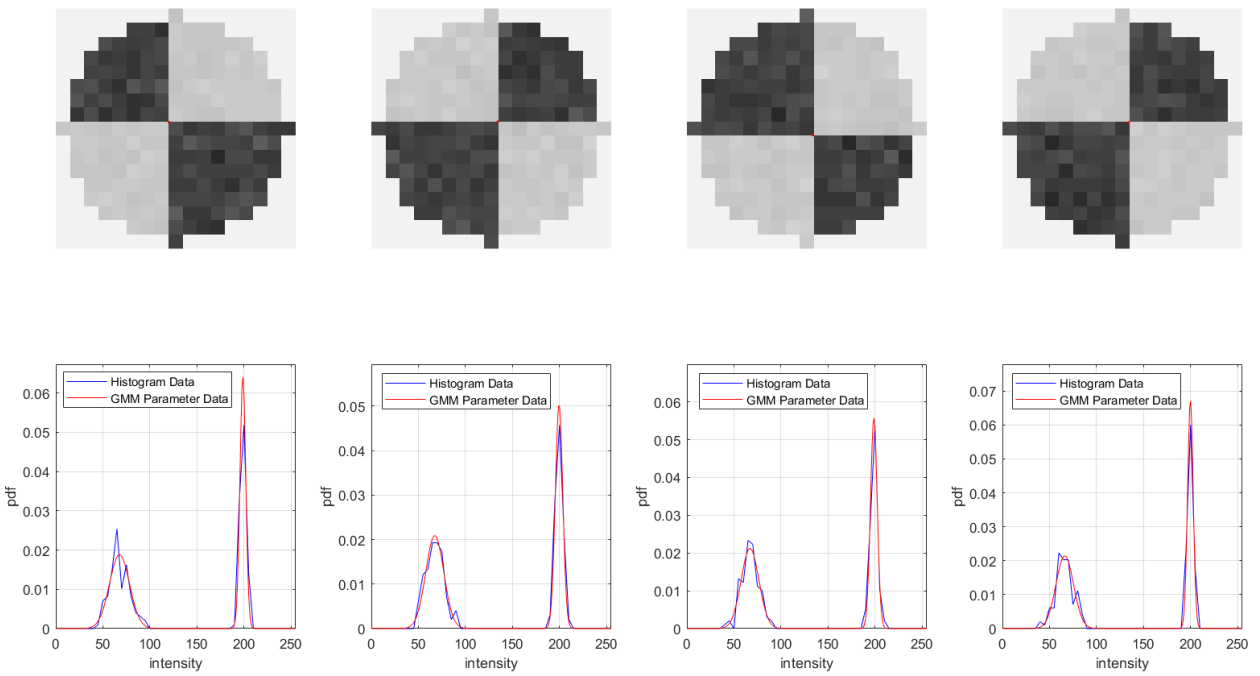

Figure 7: Gaussian mixture model fitting using simulated images
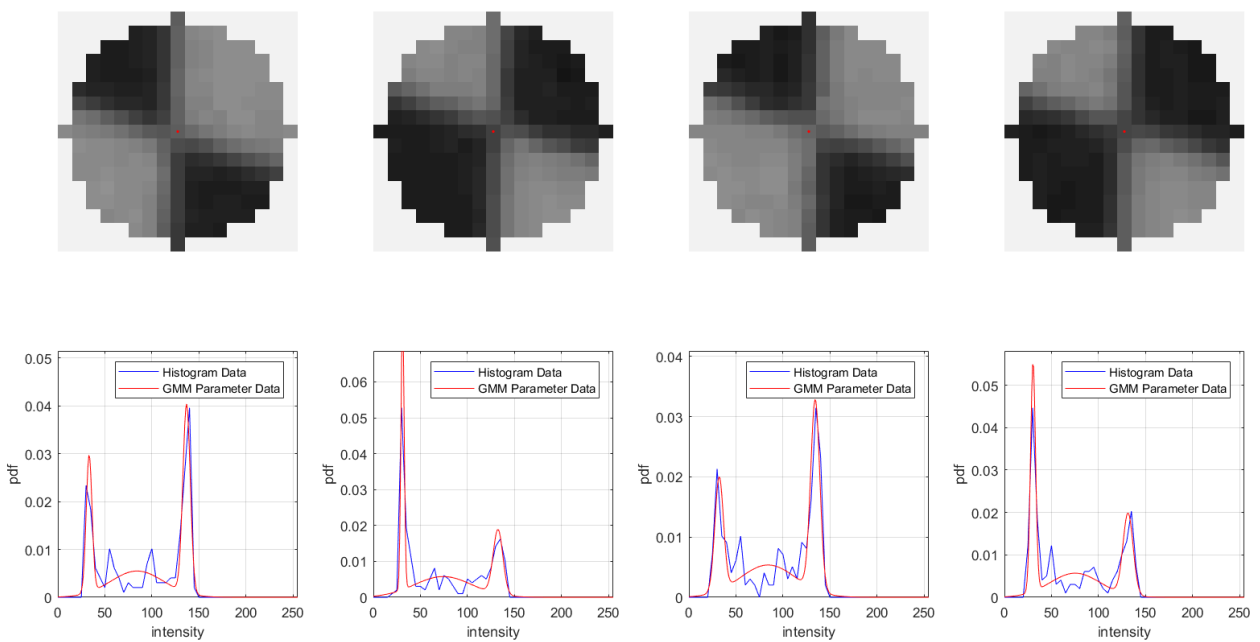

Figure 8: Gaussian mixture model fitting using real images 
This is due to spatial quantization of the pixels and the optical blurring impact from the physical lenses. In order to precisely model the geometric error of the feature extraction, the lens impact is also considered in our proposed model. According to the work from computer vision and image processing community [4], the blurring impact from the lens is usually modelled as a low-pass Gaussian filtering on the original image. The uncertainty in the extracted corner location grows increasingly with the Gaussian filter standard deviation $\sigma_{L}$. According to parameter fitting, $\sigma_{L}$ affects the feature location uncertainty as follows

$$
\sigma_{u} \sim \alpha_{1}+\alpha_{2} \sigma_{L}^{\alpha_{3}},
$$

where $\alpha_{1}, \alpha_{2}, \alpha_{3}$ are fitting coefficients for specific optical systems.

By exploiting the a priori information of the chessboard, the aperture (i.e., the covariance) of the Gaussian filter can be estimated using a maximum-likelihood (ML) estimator. We estimate the Gaussian filter aperture by first generating an ideal local patch $\tilde{I}$ around the corner located at $u$. The estimated mean values $\hat{\bar{w}}$ and $\hat{\bar{b}}$ from the GMM are applied respectively for intensity values of the white and black components of the image. Then the measurement image with lens blurring impact can be modeled as

$$
I=\tilde{I} \star G\left(\sigma_{L}\right)+n_{I},
$$

where $\star$ denotes convolution operator, $n_{I}$ the photometric noise, and $G\left(\sigma_{L}\right)$ is a two-dimensional Gaussian kernel with covariance $\sigma_{L}^{2}$. A large $\sigma_{L}$ results in more heavily blurred measurement images due to lens. Given the measurement image $I$, the optimal filter covariance using an ML estimator can be obtained by

$$
s_{L}=\hat{\sigma}_{L}=\arg \max _{\sigma_{L}} p\left(I \mid \sigma_{L}\right) .
$$

With Gaussian photometric noise assumption, the optimization in Eqn. (12) can be solved with the corresponding leastsquares estimator equivalently. We use $s_{L}$ instead of $\hat{\sigma}_{L}$ in the model for notation clarity, since the standard deviation of the parameter will also be included in the conservative modelling strategy later. As a result, all the three essential parameters for the CCGEM model $\sigma_{I}, \Delta I, s_{L}$ are extracted from the image.

For a particular camera-lens system, coefficients in Eqn. (4) can be estimated from a least squares (LS) curve fitting using all the available chessboard corners in the aforementioned offline calibration process as

$$
\left\{\hat{\alpha}_{i} \mid i=1,2,3\right\}=\arg \min _{\alpha_{i}} \sum_{j=1}^{N_{c}}\left(\left(\alpha_{1}+\alpha_{2} s_{L_{j}}^{\alpha_{3}}\right) \frac{\sigma_{I_{j}}}{\Delta I_{j}}-\varsigma_{u_{j}}\right)^{2},
$$

where $N_{c}$ is the number of chessboard corners used in calibration, and $j$ is the corresponding index. $\varsigma_{u_{j}}$ is the dispersion obtained from statistics. With the known coefficients, for a corner in an arbitrary measurement image, a prediction of the geometric error standard deviation $\sigma_{u}$ can be calculated using the proposed model by applying $\sigma_{u}=g\left(\sigma_{I}, \Delta I, s_{L}\right)$ in Eqn. (4), i.e.,

$$
\tilde{\sigma}_{u}=\left(\hat{\alpha}_{1}+\hat{\alpha}_{2} s_{L}^{\hat{\alpha}_{3}}\right) \frac{\hat{\sigma}_{I}}{\widehat{\Delta I}} \text {. }
$$

\section{CONSERVATIVE ERROR MODELLING FOR SAFETY CRITICAL APPLICATIONS}

The proposed model is able to describe the geometric error of features using only a few parameters and coefficients. Nevertheless, if the CCGEM model is used for safety critical applications such as integrity monitoring of visual navigation in practice, uncertainties in the model should be overbounded conservatively and potential risks in the parameter extraction should be considered, so that it does not cause misleading information. There are two potential risks in the aforementioned parameter extraction process:

1. Risk 1: The extracted intensity gradient $\Delta I$ is overestimated. The probability of the risk is denoted by $p\left(R_{1}\right)$.

2. Risk 2: The lens blurring factor $s_{L}$ is underestimated. The probability of the risk is denoted by $p\left(R_{2}\right)$.

The risk 1 may happen due to the impact of the existence of the optical blur in the corner extraction process. As shown in Fig. 2 and Fig. 8, the optical blur results in a gray zone at the color borders and a corresponding gray peak in the histogram. 
In the feature detection process, the applied intensity gradient measurements are not always the intensity difference between a noisy white pixel and a black pixel, but may include pixels in the gray zone. Denoting the probability of the gray peak in the Gaussian mixture distribution as $p_{g}$, the risk probability

$$
p\left(R_{1}\right)=1-\left(1-p_{g}\right)^{2 N_{g}},
$$

where $N_{g}$ is the number of border pixel pairs in the local patch used for corner extraction.

The reason of the second risk is that the estimate of $s_{L}$ has stochastic uncertainty. Since the ML estimator of $s_{L}$ uses noisy measurement image, the uncertainty of the estimated $s_{L}$ increases with the strength of the lens blurring as well as the measurement noise level. By using curve fitting, the estimation covariance $\sigma_{S_{L}}$ can be modelled as

$$
\sigma_{S_{L}}=\left(\beta_{1}+\beta_{2} s_{L}\right) \frac{\sigma_{I}}{\Delta I} .
$$

The coefficients $\beta_{1}$ and $\beta_{2}$ can be obtained in the calibration process. As a result, the lens blurring parameter $s_{L}$ can be overbounded by

$$
\check{s}_{L}=s_{L}+k_{L} \sigma_{S_{L}}
$$

with confidence level dependent on the parameter $k_{L}$. The risk probability $p\left(R_{2}\right)$ is the double-sided tail probability corresponding to a given expand factor $k_{L}$.

Moreover, since the uncertainty of the data used for calibration increases with the image noise, a least-squares fitting of the coefficients in Eqn. (13) is not able to ensure that the modeled error overbounds the actual error with a given confidence level. In order to have a conservative model overbounding the error for integrity of safety-critical applications, a conservative coefficients fitting strategy which overbounds all the training samples should be applied. By inflating the coefficients until all the samples are overbounded, the conservative coefficients can be obtained as $\left\{\check{\alpha}_{i} \mid i=1,2,3\right\}$. As a result, for integrity monitoring or other safety-critical applications, the corner location error can be overbounded by a Gaussian model with standard deviation $\check{\sigma}_{u}$

$$
\check{\sigma}_{u}=\left(\check{\alpha}_{1}+\check{\alpha}_{2} \check{s}_{L}^{\check{\alpha}_{3}}\right) \frac{\sigma_{I}}{\Delta I} .
$$

The geometric error model obtained from both LS fitting and conservative fitting are shown in Fig. 9 as the blue surface and the green surface, respectively. 

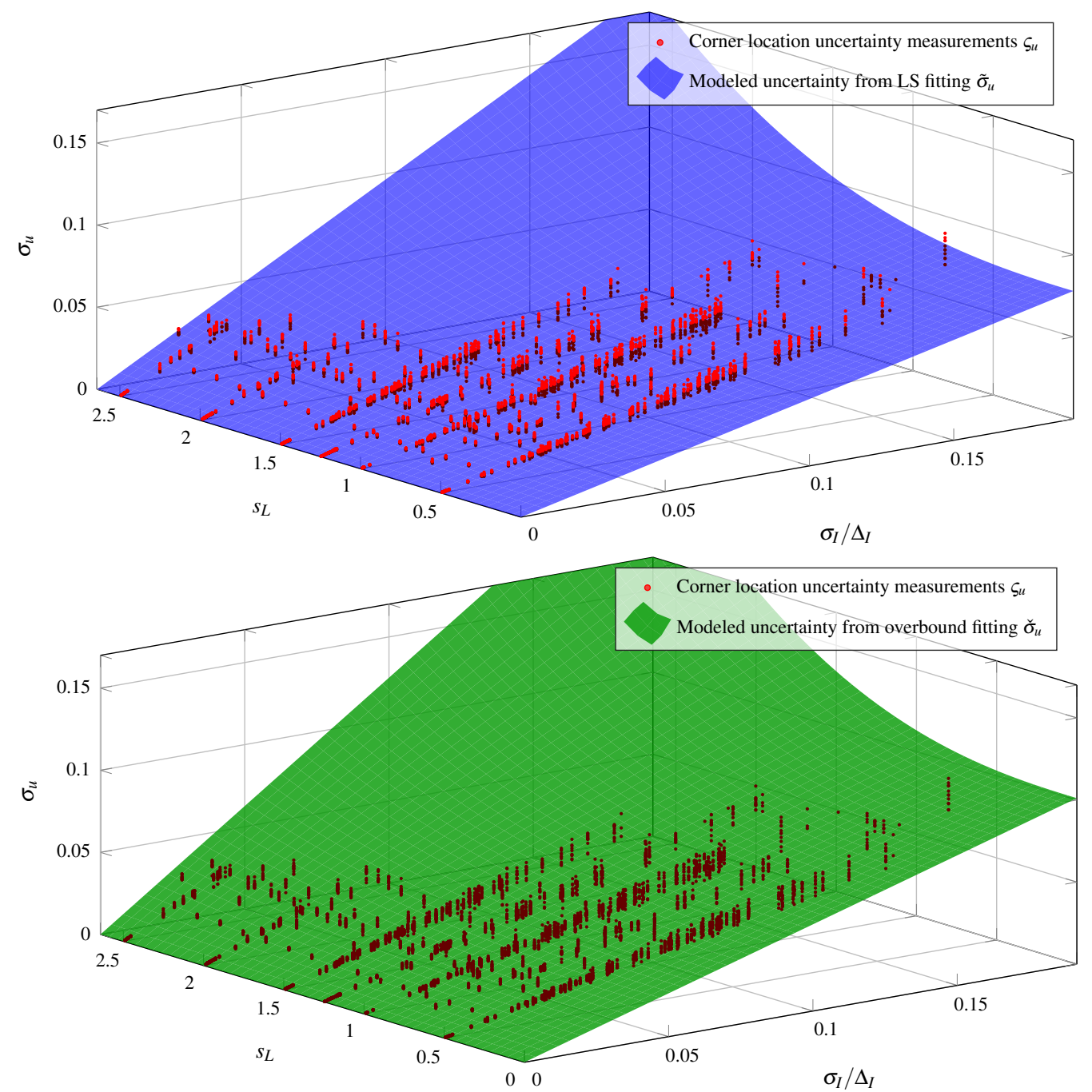

Figure 9: Uncertainty model fitting with respect to $\sigma_{I}, \Delta_{I}$ and $s_{L}$ : the LS fitting and the overbound fitting

Each dot in the plots denotes the location uncertainty of a corner sample used in calibration for the coefficients fitting. It can be seen that as the image noise increases, besides the inclination of the corner location error $\sigma_{u}$, the dispersion of $\varsigma_{u}$ from the calibration images also increases. It can be observed that the uncertainty measurements from some corners are larger than the values of the blue surface, which represents the uncertainty model obtained from the LS fitting. Meanwhile, the green surface obtained from the conservative fitting strategy ensures that all the sample points are overbounded in the fitting process. The difference can be better illustrated by the side view in Fig. 10. 

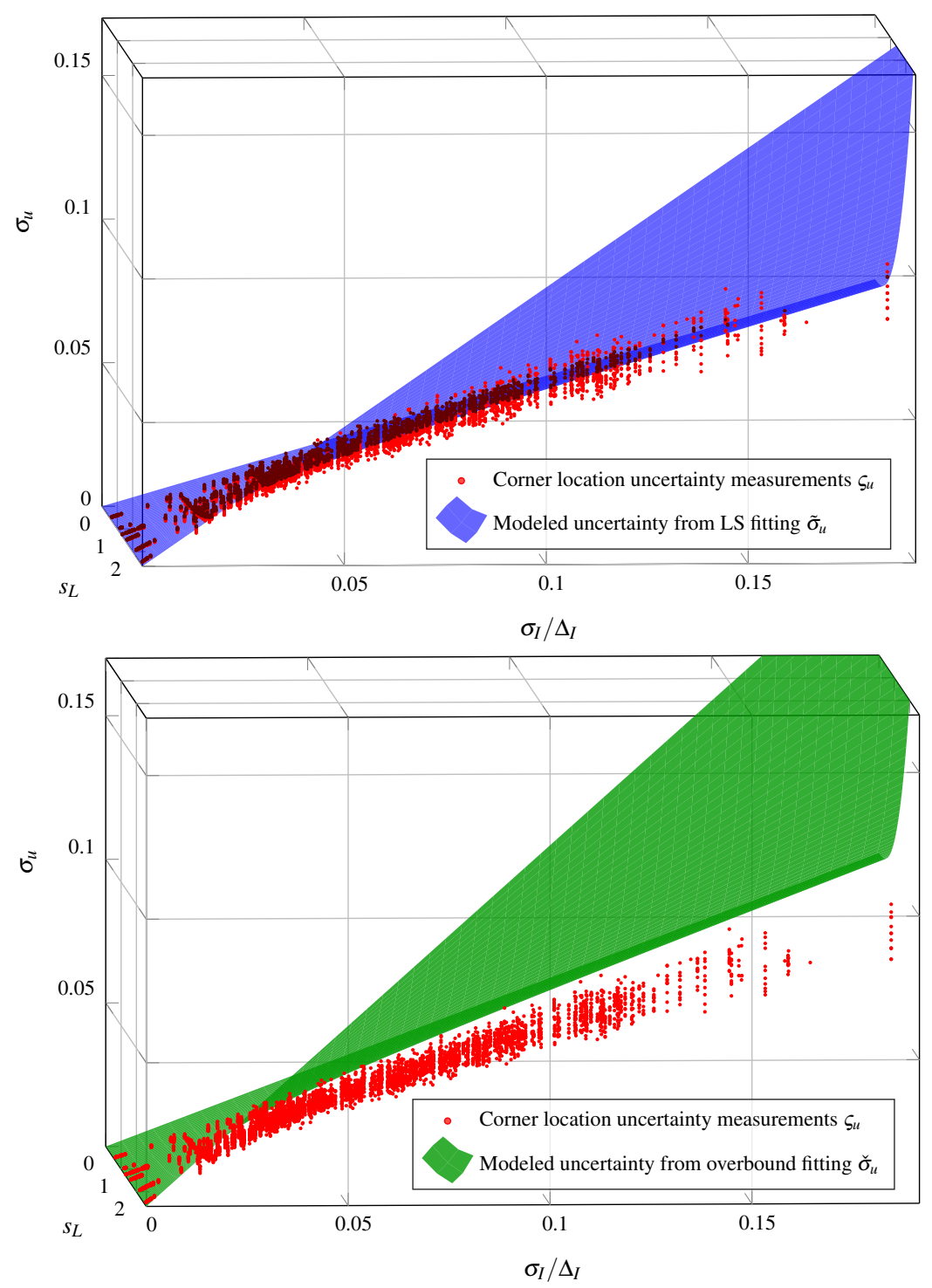

Figure 10: Uncertainty model fitting with respect to $\sigma_{I}, \Delta_{I}$ and $s_{L}$ (side view)

\section{EXPERIMENTAL RESULTS OF APPLYING CCGEM}

First of all, we verify the correctness of the CCGEM model in Eqn. (4) using the test images. Fig. 11 shows the standard deviation of the feature location error with respect to different parameters as examples.

The feature location uncertainty of $x$ and $y$ dimensions from the test images are plotted as red dots. The curve fitting results for different local parameters $\left(\Delta I, \sigma_{I}, s_{L}\right)$ are shown in blue in the plots. First, it has verified that the error distribution in the two dimensions are isotropic. The left and middle plots have the same $\sigma_{I}$ value, but the ratio of $\sigma_{I}$ to $\Delta I$ is twice as large in the left plot scenario as in the middle one. It can be seen that the geometric error covariance is also twice as large. Meanwhile, the left and right plots have the same $\sigma_{I} / \Delta I$ ratio, but the two individual parameters are different. It can be observed that in such case, the geometric error covariances in the two scenarios are the same. Therefore, it can be verified that the geometric error is proportional to the factor $\sigma_{I} / \Delta I$, which is predicted by the theory. In addition, in all the three plots, the geometric error follows the same function with respect to the lens blur parameter $s_{L}$. It can be concluded that the proposed CCGEM model properly describes the geometric error change with respect to the parameters.

We further verify and analyze the model performance by using different real test images. Our test data set consists of 12 scenarios with 2000 images each. In each image, 48 chessboard corners are extracted. The geometric error uncertainty $\varsigma_{u}$ can be calculated from the statistics of the 2000 images for each individual corner. As we stated as a challenge in the 

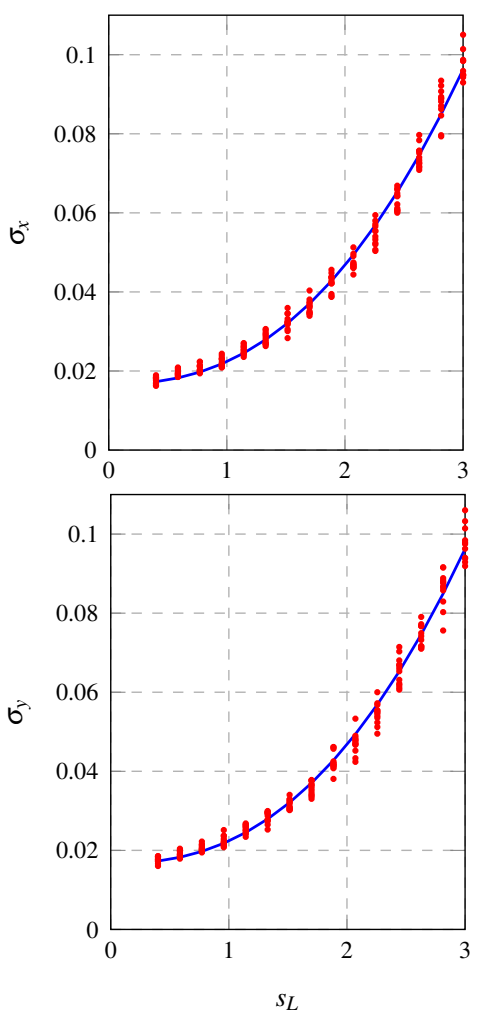

(a)

$$
\begin{aligned}
\Delta_{I} & =0.35 \\
\sigma_{I} & =0.0141 \\
\sigma_{I} / \Delta_{I} & =0.0404
\end{aligned}
$$
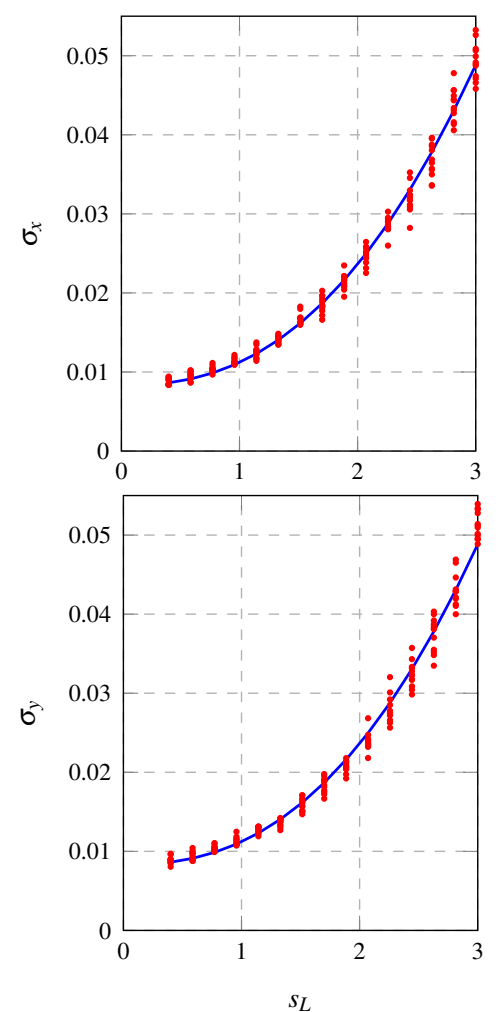

(b)

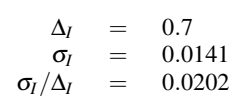

Figure 11: Model verification
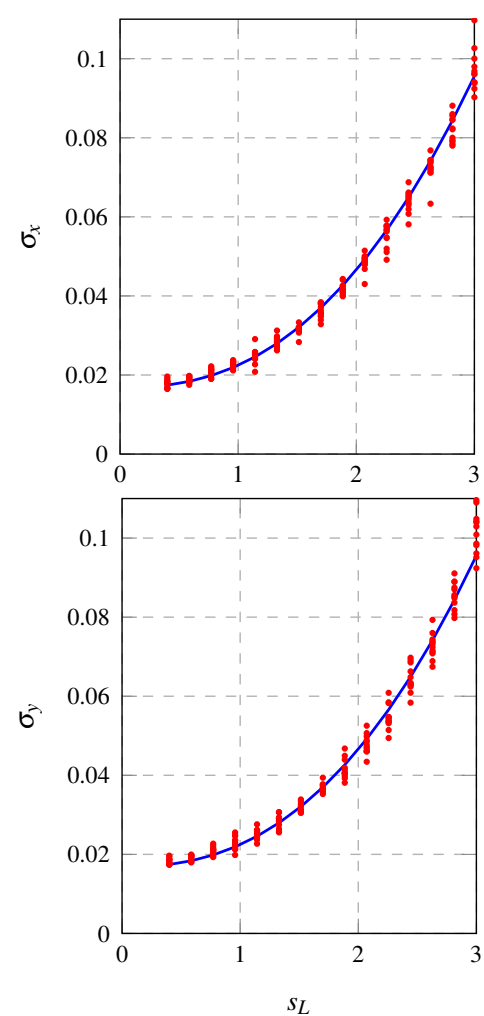

(c)

introduction, the local lighting conditions for all corner are slightly different, which results in different error distributions. In order to properly demonstrate the CCGEM performance, we plot the distribution of the geometric error $\varepsilon_{x}$ and $\varepsilon_{y}$ normalized by the model predicted standard deviation $\tilde{\sigma}_{u}$. Ideally, if the model perfectly describes the error distribution, i.e., $\tilde{\sigma}_{u}=\sigma_{u}$, the ratio $\varepsilon_{x} / \tilde{\sigma}_{u}$ and $\varepsilon_{y} / \tilde{\sigma}_{u}$ will follow a standard Gaussian distribution. For the utilized test images dataset, the histogram of the corner geometric error normalized by modelled standard deviation is shown in Fig. 12. It can be observed that the histogram approximately follows a standard normal distribution, which indicates that the predicted error by CCGEM well describes the stochastic error of the feature location.

Nevertheless, it can be observed from Fig. 12 that at the tails of the distribution, the standard normal distribution does not always overbound the normalized error. To better illustrate the tails part, the Quantile-Quantile plot (QQ-plot) of the two distributions is provided in Fig. 13. It can be clearly seen that the standard normal distribution does not overbound the error tails. This indicates that in some cases with large feature geometric error, the model underestimates the error, which can be hazardous in safety critical applications such as navigation integrity monitoring.

Consequently, for safety critical applications, the aforementioned conservative error model from CCGEM should be applied instead of the least squares fitting model. Fig. 14 shows the QQ-plot of the normalized error using the conservative model versus a standard Gaussian distribution. It can be seen that the standard Gaussian distribution overbounds the error distribution tails, which indicates that the conservative model from CCGEM does not underestimate the feature location error in all the test images.

\section{CONCLUSION}

We proposed a geometric error model named CCGEM for chessboard-like visual features (' $\mathrm{X}$ '-junctions). The model describes the extracted feature location error distribution as a function of several local parameters around the corner. CCGEM can be applied in different lighting conditions and with different optical systems. The coefficients of the model can be obtained in an offline calibration process. CCGEM provides a least squares fitting model and a conservative model for 


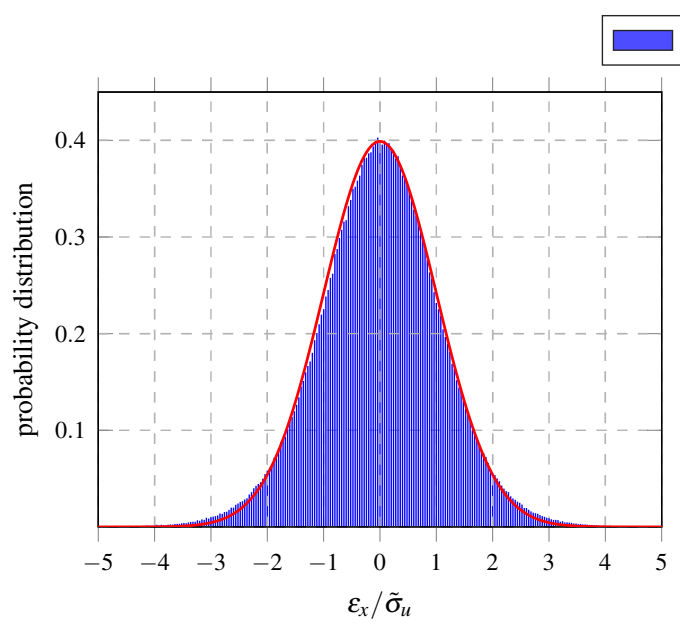

(a) Horizontal error

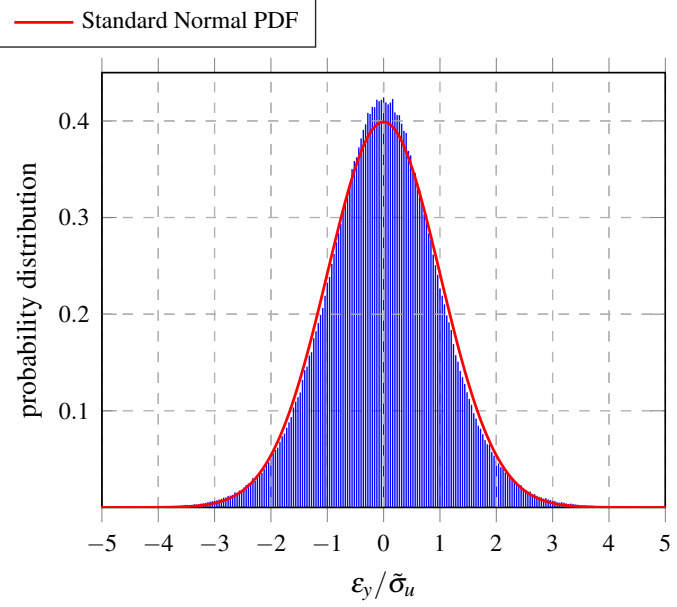

(b) Vertical error

Figure 12: Modeled Uncertainty Check: Error Histogram Comparison to Standard Normal Distribution
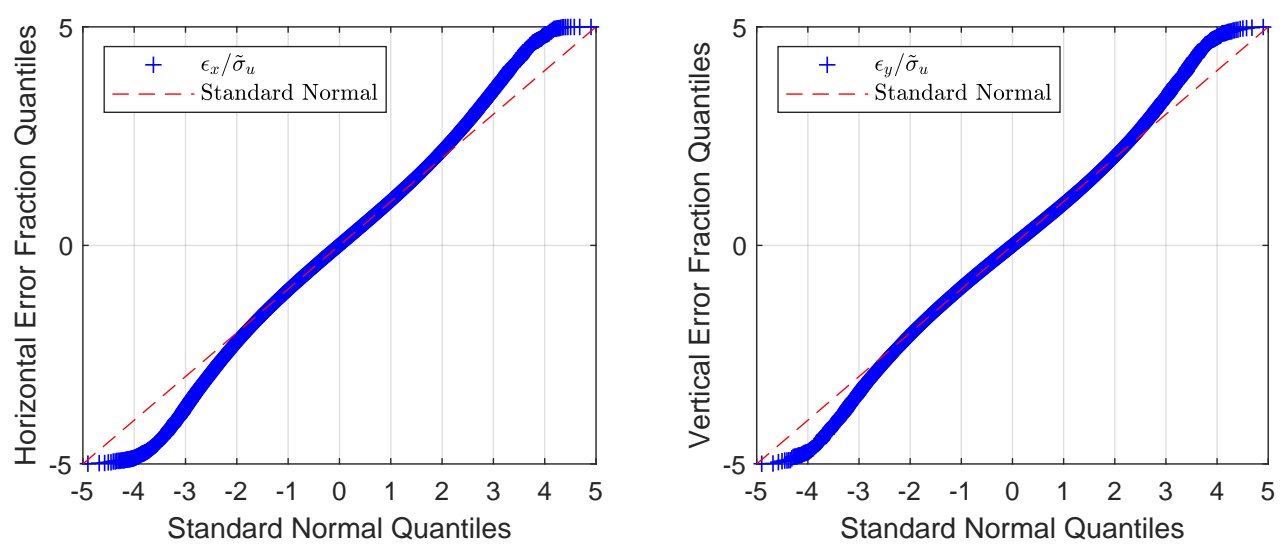

Figure 13: Quantile-Quantile plot for model using least squares fitting
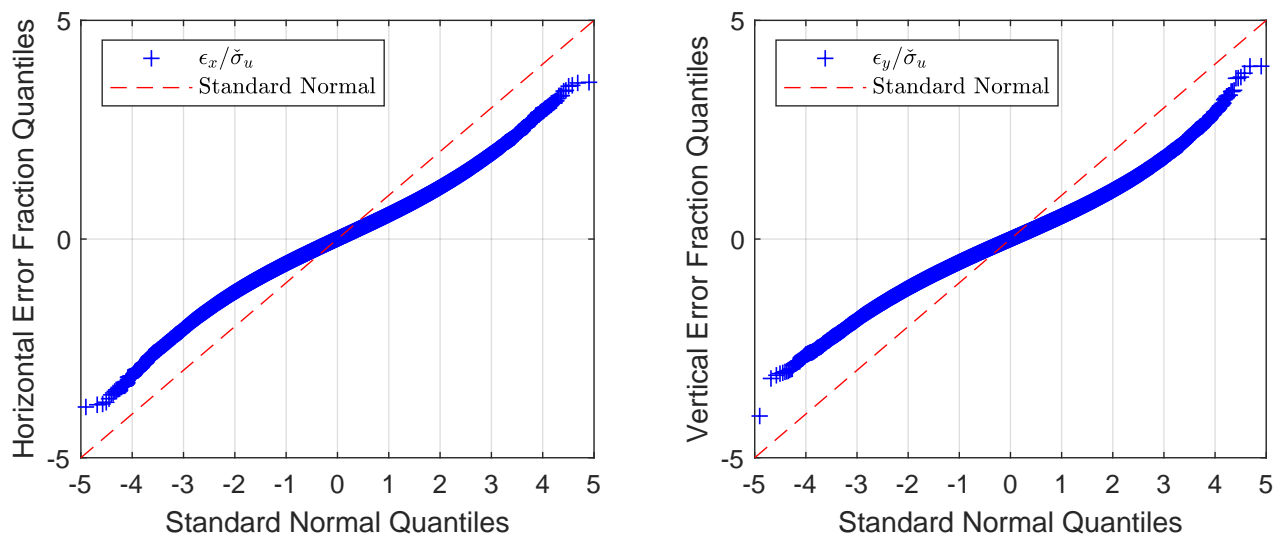

Figure 14: Quantile-Quantile plot for model using conservative fitting 
safety critical applications at the same time. The least squares fitting model predicts the measurement error distribution well. Meanwhile, the conservative model ensures that the model overbounds the large measurement error for not generating misleading information. 


\section{REFERENCES}

[1] C. S. Sharp, O. Shakernia, and S. S. Sastry, "A vision system for landing an unmanned aerial vehicle," in Proceedings 2001 ICRA. IEEE International Conference on Robotics and Automation (Cat. No. 01CH37164), vol. 2. Ieee, 2001, pp. 1720-1727.

[2] A. Cesetti, E. Frontoni, A. Mancini, P. Zingaretti, and S. Longhi, "A vision-based guidance system for uav navigation and safe landing using natural landmarks," Journal of Intelligent and Robotic Systems, vol. 57, no. 1, p. 233, Oct 2009. [Online]. Available: https://doi.org/10.1007/s10846-009-9373-3

[3] L. Narula, J. M. Wooten, M. J. Murrian, D. M. LaChapelle, and T. E. Humphreys, "Accurate collaborative globally-referenced digital mapping with standard gnss," Sensors, vol. 18, no. 8, 2018. [Online]. Available: https://www.mdpi.com/1424-8220/18/8/2452

[4] R. Jain, R. Kasturi, and B. G. Schunck, Machine vision. McGraw-Hill New York, 1995, vol. 5.

[5] R. Mur-Artal and J. D. Tards, "Orb-slam2: An open-source slam system for monocular, stereo, and rgb-d cameras," IEEE Transactions on Robotics, vol. 33, no. 5, pp. 1255-1262, Oct 2017.

[6] R. Hartley and A. Zisserman, Multiple view geometry in computer vision. Cambridge university press, 2003.

[7] R. Kumar and O. Osechas, "Integrity Potential of Landmark-Based Navigation," in Proc. of the Global Nav. Sat. Systems Conf. of the ION. Miami, FL, 2018.

[8] M. J. Edwards, M. P. Hayes, and R. D. Green, "Error distribution of estimated checkerboard corner location," in 2018 International Conference on Image and Vision Computing New Zealand (IVCNZ), Nov 2018, pp. 1-6.

[9] L. Lucchese and S. K. Mitra, "Using saddle points for subpixel feature detection in camera calibration targets," in Asia-Pacific Conference on Circuits and Systems, vol. 2, Oct 2002, pp. 191-195 vol.2. 